\title{
Agent based Congestion Control Performance in Mobile ad-hoc Network:A Survey paper
}

\author{
Vishnu Kumar Sharma \\ Department of CSE, \\ JUET,India
}

\author{
Dr. Sarita Singh Bhadauria \\ Department of Elex, \\ MITS,India
}

\begin{abstract}
A Congestion control is a key problem in mobile adhoc networks. The standard TCP congestion control mechanism is not able to handle the special properties of a shared wireless channel. Many approaches have been proposed to overcome these difficulties. ideas and show their interrelations. mobile agent based congestion control Technique routing is proposed to avoid congestion in ad hoc network. Some mobile agents are added in ad hoc network, which carry routing information and nodes congestion status. When mobile agent travels through the network, it can select a less-loaded neighbor node as its next hop and update the routing table according to the node's congestion status. With the aid of mobile agents, the nodes can get the dynamic network topology in time. In this paper, we give an overview over existing proposals, explain their key ideas, TCP Issues, Reduce the Congestion, delay in mobile ad-hoc network and proposed solution
\end{abstract}

Keywords- Mobile Ad hoc Networks (MANETs); mobile agents (MA); TCP.

\section{INTRODUCTION}

An ad hoc network is also called as infrastructure less networks which is a collection of mobile nodes which forms a temporary network without the help of central administration or standard support devices regularly available in conventional networks. Mobile ad hoc wireless networks have the ability to establish networks at anytime, anywhere to possess the assurance of the future. These networks do not depend on irrelevant hardware because it makes them ideal candidate for rescue and emergency operations. The constituent wireless nodes of these network build, operate and maintain these networks. Each node asks the help of its neighboring nodes to forward packets because these nodes usually have only a limited transmission range [1].

Congestion occurs in mobile ad hoc networks (MANETs) with limited resources. In such networks, packet transmissions suffer from interference and fading, due to the shared wireless channel and dynamic topology. Congestion leads to packet losses and bandwidth degradation, and wastes time and energy on congestion recovery. A congestion-aware routing protocol can preempt congestion through bypassing the affected links [2]. Several distinct congestion-related problems have been identified and tracked down, including severe throughput degradation and massive fairness problems. They have been shown to originate from the MAC, routing, and transport layers [4].
TCP congestion control works very well on the Internet. But MANETs exhibit some unique properties that greatly affect the design of appropriate protocols and protocol stacks in general, and of congestion control mechanism in particular. As it turned out, the vastly differing environment in a mobile adhoc network is highly problematic for standard TCP.

- Route failures trigger inappropriate TCP congestion control reactions.

- The standard TCP retransmission timeout grows too fast in MANET environments.

- The locally shared medium induces unfairness between TCP flows.

- TCP has a long feedback path.

- Data and acknowledgment packets interfere on the shared medium.

- TCP over saturates the network.

- On the shared medium, there is intra-flow contention between successive data packets.

- The TCP acknowledgment scheme generates a lot of packets.

- TCP traffic is bursty.

- TCP's basic design decisions do not fit a MANET environment well. [4]

TCP congestion control has an implicit assumption, which is that any packet loss is due to network congestion. However, this assumption is no longer valid in the MANET as packet losses may well be due to channel bit errors, medium contention, and route failures. [14]

Congestion is a major cause for packet loss in MANETs and reducing packet loss involves congestion control running on top of a mobility and failure adaptive routing protocol at the network layer. Congestion non-adaptive routing in MANETs may lead to the following problems:

- Long delay: It takes time for a congestion to be detected by the congestion control mechanism. In severe congestion situations, it may be better to use a new route. The problem with an on-demand routing protocol is the delay it takes to search for the new route.

- High overhead: In case a new route is needed, it takes processing and communication effort to discover it. If multipath routing is used, though an alternate route is readily found, it takes effort to maintain multiple paths. 
- Many packet losses: Many packets may have already been lost by the time congestion is detected. A typical congestion control solution will try to reduce the traffic load, either by decreasing the sending rate at the sender or dropping packets at the intermediate nodes or doing both. The consequence is a high packet loss rate or a small throughput at the receiver [3].

Flow control is a good mechanism to avoid the congestion problem. But it is another major challenge in the network research that adapts the transmission rate to the available resources capacities in order to avoid congestion [13].

\section{RELATED WORK}

This section presents a brief review of the work already done in this field.

Kazuya Nishimura et al [5] have discussed a routing protocol that uses multi-agents to reduce network congestion for a Mobile Ad hoc Network (MANET). They have extended a dynamic routing protocol using mobile agent's protocol to be more generic, so that it can be effective in the face of network congestion. They have developed both simulation environment and protocols, and performed simulations under different conditions of mobility and traffic patterns to demonstrate the effectiveness of their approach.

Yao-Nan Lien et al [6] proposed a new TCP congestion control mechanism by router-assisted approach. Based on the information feed backed from routers, a TCP sender is able to adjust its sending speed dynamically in order to avoid overshooting problem.

Wei Sun et al [7] have compared the general AIMD-based congestion control mechanism (GAIMD) with Equation-based congestion control mechanism (TFRC TCP-Friendly Rate Control) over a wide range of MANET scenario, in terms of throughput fairness and smoothness. Their results have shown that TFRC and GAIMD are able to maintain throughput smoothness in MANET, but at the same time, they require only a less throughput than the competing TCP flows. Also their results show that TFRC changes its sending rate more smoothly than GAIMD does, but it gets the least throughput compares with TCP and GAIMD.

Consolee Mbarushimana et al [8], have exposed the performance of MANETs routing protocols is highly dependent on the type of traffic generated or routed by intermediate nodes. They have proposed a Type of Service Aware routing protocol (TSA), an enhancement to AODV, which uses both the ToS and traditional hop count as route selection metrics. TSA avoids congestion by distributing the load over a potentially greater area and therefore improving spatial reuse. Their simulation study reveals that TSA considerably improves the throughput and packet delay of both low and high priority traffic under different network operational conditions.

Yung Yi et al [9] have developed a fair hop-by-hop congestion control algorithm with the MAC constraint being imposed in the form of a channel access time constraint, using an optimization-based framework. In the absence of delay, they have shown that their algorithm is globally stable using a Lyapunov-function-based approach. Next, in the presence of delay, they have shown that the hop-by-hop control algorithm has the property of spatial spreading. Also they have derived bounds on the "peak load" at a node, both with hop-by-hop control, as well as with end-to-end control, show that significant gains are to be had with the hop-by-hop scheme, and validate the analytical results with simulation.

Umut Akyol et al [10] have studied the problem of jointly performing scheduling and congestion control in mobile adhoc networks so that network queues remain bounded and the resulting flow rates satisfy an associated network utility maximization problem. They have defined a specific network utility maximization problem which is appropriate for mobile adhoc networks. They have described a wireless Greedy Primal Dual (wGPD) algorithm for combined congestion control and scheduling that aims to solve this problem. They have shown how the wGPD algorithm and its associated signaling can be implemented in practice with minimal disruption to existing wireless protocols.

S.Karunakaran et al [11] have presented a Cluster Based Congestion Control (CBCC) protocol that consists of scalable and distributed cluster-based mechanisms for supporting congestion control in mobile ad hoc networks. The distinctive feature of their approach is that it is based on the selforganization of the network into clusters. The clusters autonomously and proactively monitor congestion within its localized scope.

Kazuya Nishimura et al [12] have discussed a routing protocol that uses multi-agents to reduce network congestion for MANET. In their work, two kinds of agents are engaged in routing. One is a Routing Agent that collects information about network congestion as well as link failure. The other is a Message Agent that uses this information to get to their destination nodes.

\section{THE PROPOSED WORK}

Congestion adaptive routing has been investigated in several studies as we explained in section 2. The approaches in all the cited studies converge in evaluating or assessing the level of activity in intermediate nodes by measuring either the load or the delay. Based on the gathered information, the optimal path is established trying to avoid the already or likely to become congested nodes. However, none of the research reported has evaluated the effect service type of the traffic carried by intermediate nodes has on the performance of routing protocols [8].

The route discovery process of most of MANETs routing protocols do not consider the status of their queues, before advertise themselves as candidate to route traffic to the destination. This might result into long delays or packet drops for newly arriving traffic, failing to be transmitted ahead of the already queuing traffic [8].

The performance of the mobile ad hoc networks is strongly influenced by the congestion problem. A congestion control scheme consists of a routing algorithm and a flow control scheme. In earlier research, the routing and the flow control problems have been considered separately. To achieve better 
performance and better congestion control, the routing and the flow control must be considered jointly [13].

In this paper, we propose to design and develop an agent based congestion control architecture in fig.-1, In this architecture, all the nodes are mobile and information about network congestion is collected and distributed by mobile agents (MA). Each node has a routing table that stores route information for every destination. MA starts from every node and moves to an adjacent node at every time. The MA updates the routing table of the node it is visiting.

In this proposal, the node is classified in one of the four categories depending on whether the traffic belongs to background, best effort, video or voice AC respectively. Then MA at each node estimates the congestion level for each traffic class by checking the queue status and a priority is assigned for the node based on the measured congestion level. Using this classification, a node with no traffic or with delay-insensitive traffic is considered more priority so that it can receive more traffic than a low priority node. The congestion level of every node is updated every time there is change in traffic type, and it is periodically propagated to neighbors.

\section{Agent BAsed Congestion CONTROL Routing}

The agent based congestion routing can be explained from the following figure:

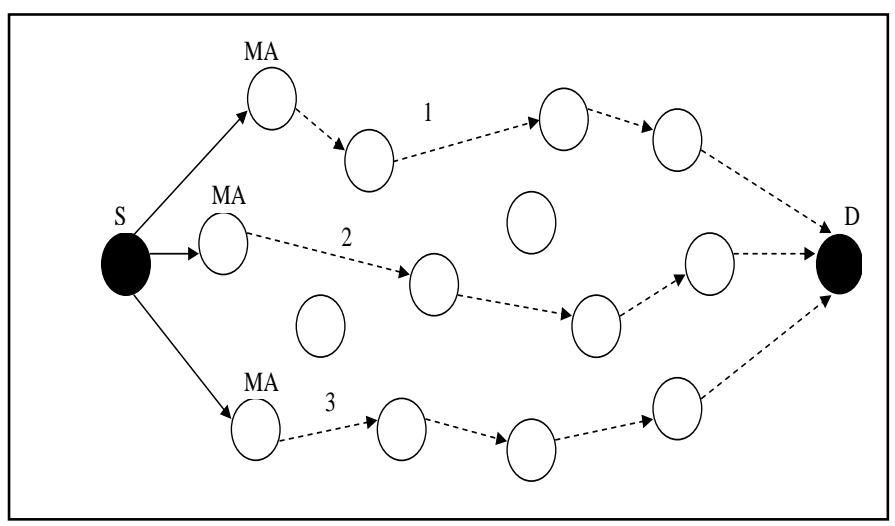

Figure 1. Agent Based Congestion Routing

Step 1: The source $\mathrm{S}$ checks the number of available one hop neighbors and clones the Mobile Agent (MA) to that neighbors.

Step 2: The Mobile Agent selects the shortest path of the route to move towards the destination $\mathrm{D}$ as given in the figure 1 such as P1, P2 and P3.

Step 3: The MA1 moves towards the destination D in a hopby-hop manner in the path P1 and MA2 in P2 and MA3 in P3 respectively.

Step 4: Then the MA1 calculates the Total Congestion Metric(TCM), TCM1 of that path P1 and similarly MA2 calculates the TCM2 of P2 and MA3 calculates the TCM3 of P3.
Step 5: Now the destination D sends the total congestion metrics TCM1, TCM2 and TCM3 of the paths P1, P2 and P3 respectively to the source.

Step 6: Now the source selects path using min (TCM1, TCM2, and TCM3) and sends the data through the corresponding path which has the minimum congestion.

\section{Mobile Agents TeChNiQue}

Mobile agents are software entities that act on behalf of their creators and move independently between hosts. In general, a mobile agent executes on a machine that hopefully provides the resources or services that it needs to do its work. If a machine does not contain the needed resources or services, the mobile agent can transfer itself to a new machine. Lange and Oshima [15] enumerate several beets of using mobile

agents. Of particular interest to MANET routing are:

- Mobile agents are able to upgrade protocols in use by moving to a destination and setting up communications operating under revised policies.

- After being dispatched, mobile agents become indecentdent of the process that created them and can operate asynchronously and react dynamically and autonomously to environmental changes.

- Mobile agents can reduce network load and latency by running remotely.

Recently, a number of mobile agent systems have been developed to address applications in areas including telecommunication services, E-commerce and personal assistance. Included among these are Agent TCL [16] (later D'Agents),ARA [17], Concordia [18], and Aglets [19]. All such systems provide common functions including agent migration, inter-agent communication and security. One potential drawback of using mobile agents is that the agents require an "execution environment" in which to run. This has become less of an issue in recent years as mobile devices become more capable and the execution environments become somewhat leaner.

\section{A. Routing Using Mobile Agents}

Early work on routing in dynamic networks using mobile agents by Kramer et al. [20] concentrated on route discovery using agents to continuously track the network topology and update routing tables at all mobile hosts reached. When a route is requested, an agent is sent to discover routes to the destination.

These agents analyze the routing tables on the hosts they arrive at and either return a discovered route to the sender or move on to another machine if no route is found. Unfortunately, this method increases network load

\section{B. Mobile Agent Technology}

Mobile Agent is a novel way of building distributed software system. Traditional distributed systems are built out of 
stationary programs that pass data back and forth across a network [21]. It is usually kept a certain state. It is able to exchange information for its owners and other nodes in order to work together.

\section{CONCLUSION}

In this paper, we have discussed an agent based congestion control technique and TCP issues. In our technique, the information about network congestion is collected and distributed by mobile agents (MA). A mobile agent starts from every node and moves to an adjacent node at every time. A node visited next is selected at the equivalent probability. The MA brings its own history of movement and updates the routing table of the node it is visiting. The MA updates the routing table of the node it is visiting. In this technique, the node is classified in one of the four categories depending on whether the traffic belongs to background, best effort, video or voice AC respectively. our proposed technique attains high delivery ratio and throughput with reduced delay when compared with the existing technique

\section{REFERENCES}

[1] S.Santhosh baboo and B.Narasimhan, "A Hop-by-Hop CongestionAware Routing Protocol for Heterogeneous Mobile Ad-hoc Networks", International Journal of Computer Science and Information Security, 2009

[2] Xiaoqin Chen, Haley M. Jones and A.D.S. Jayalath, "Congestion-Aware Routing Protocol for Mobile Ad Hoc Networks", IEEE $66^{\text {th }}$ Conference in Vehicular Technology, 2007.

[3] Duc A. Tran and Harish Raghavendra, "Congestion Adaptive Routing in Mobile Ad Hoc Networks", IEEE Transactions on Parallel and Distributed Systems, November 2006

[4] Christian Lochert, Bjorn Scheuermann and Martin Mauve, "A Survey on Congestion Control for Mobile Ad-Hoc Networks", Wireless Communications and Mobile Computing, InterScience, 2007.

[5] Kazuya Nishimura and Kazuko Takahashi, "A Multi-Agent Routing Protocol with Congestion Control for MANET", Proceedings $21 \mathrm{st}$ European Conference on Modeling and Simulation, 2007

[6] Yao-Nan Lien and Ho-Cheng Hsiao, "A New TCP Congestion Control Mechanism over Wireless Ad Hoc Networks by Router-Assisted Approach", 27th IEEE International Conference on Distributed Computing Systems Workshops, 2007.

[7] Wei Sun, Tao Wen and Quan Guo, "A Performance Comparison of
Equation-Based and GAIMD Congestion Control in Mobile Ad Hoc Networks", International Conference on Computer Science and Software Engineering, 2008

[8] Consolee Mbarushimana and Ali Shahrabi, "Congestion Avoidance Routing Protocol for QoS-Aware MANETs", Proceedings of IEEE International Wireless Communications and Mobile Computing Conference, 2008.

[9] Yung Yi and Sanjay Shakkottai, "Hop-by-Hop Congestion Control Over a Wireless Multi-Hop Network", IEEE/ACM Transactions on Networking, February 2007

[10] Umut Akyol, Matthew Andrews, Piyush Gupta, John Hobby, Iraj Saniee and Alexander Stolyar, "Joint Scheduling and Congestion Control in Mobile Ad-Hoc Networks", Proceedings of IEEE INFOCOM, 2008.

[11] S.Karunakaran \& P.Thangaraj, "A CLUSTER BASED CONGESTION CONTROL PROTOCOL FOR MOBILE ADHOC NETWORKS”, International Journal of Information Technology and Knowledge Management, July-December 2010, Volume 2, No. 2, pp. 471-474.

[12] Kazuya NISHIMURA and Kazuko TAKAHASHI, "A Multi-Agent Routing Protocol with Congestion Control for MANET", Proceedings 21st European Conference on Modelling and Simulation ,2007.

[13] Belkadi Malika, Lalam Mustapha, M'zoughi Abdelaziz, Tamani Nordine, Daoui Mehammed, Aoudjit Rachida, "Intelligent Routing and FloControl In MANETs", Journal of Computing and Information Technology, doi:10.2498/cit.1001470

[14] Hongqiang Zhai, Xiang Chen and Yuguang Fang, "Rate-Based Transport Control for Mobile Ad Hoc Networks", Proceedings of IEEE WCNC'05.

[15] D. Lange and M. Oshima: "Seven Good Reasons for Mobile Agents". Communications of the ACM 42(3) (1999) 88-89.

[16] R. Gray: "Agent Tcl: A flexible and secure mobile agent system". In Proceedings of the 4th Annual Tcl/Tk workshop. Monterey, USA,July 1996, pp. 9-23.

[17] H. Peine and T. Stolpmann: "The Architecture of the Ara Platform for Mobile Agents". In Proceedings of the 1st International Workshop on Mobile Agents. Berlin, Germany, April 1997, pp. 50-61.

[18] D. Wong, N. Paciorek, T. Walsh, and J. DiCelie: "Concordia: An Infrastructure for Collaborating Mobile Agents". In Proceedings of the 1 st International Workshop on Mobile Agents. Berlin, Germany,April 1997, pp. 86-97.

[19] D. Lange, M. Oshima, G. Karjoth, and K. Kosaka: "Aglets: Programming Mobile Agents in Java". In Proceedings of Worldwide Computing and Its Applications. Tsukuba, Japan, March 1997, pp.253266.

[20] K. Kramer, N. Minar, and P. Maes:"Mobile Software Agents from Dynamic Routing”.Mobile Computing and Communications Review 3(2) (1999) 12-16.

[21] WOOK C, SAJAL K D, LEE I. Nomadic Control Packet- Based Dynamic Route Maintenance Scheme for Adaptive Routing in Mobile Ad Hoc Networks[EB/OL]. http:/l csdl.computer.org/comp/proceedings/len /2003/ 20 37/00/20370140abs.html. 